Hadron Structure '15

International Journal of Modern Physics: Conference Series

Vol. 39 (2015) 1560088 (7 pages)

(C) The Author(s)

DOI: $10.1142 /$ S2010194515600885

\title{
Heavy baryons with strangeness in soliton models
}

\author{
H. Weigel and J. P. Blanckenberg \\ Physics Department, Stellenbosch University \\ Matieland 7602, South Africa
}

Published 26 November 2015

\begin{abstract}
We present some recent results from soliton model calculations for the spectrum of baryons with a single heavy quark. The model comprises chiral symmetry for light flavors and (approximate) heavy spin-flavor symmetry for the heavy quarks. We focus on flavor symmetry breaking for strangeness degrees of freedom.
\end{abstract}

Keywords: Chiral soliton; heavy quark symmetry; flavor symmetry breaking.

PACS Numbers: 11.30.Rd, 12.39.Dc, 12.39.Hg, 14.20.-c

\section{Introduction}

Baryons containing heavy quarks have drawn renewed attention since the potential discovery of pentaquark states with hidden charm. ${ }^{1}$ Here we present a model calculation for the spectrum of baryons with a single heavy quark.

We start from a chiral soliton model with pseudoscalar and vector meson fields that reasonably well describes the spectrum, static properties and meson nucleon scattering. These studies are reviewed in Ref. 2. We then augment this model by coupling mesons with a single heavy quark (charm or bottom). This coupling is constructed to reproduce the heavy spin-flavor symmetry as the mass of the heavy meson is sent to infinity. ${ }^{3}$ The soliton then produces an attractive potential for the heavy mesons and the resulting bound states are central to the investigation of heavy baryons. ${ }^{4}$ In this presentation, which is mainly based on Ref. 5, we particularly discuss how flavor symmetry breaking between the light non-strange and strange quarks is included when coupling the chiral soliton and the heavy meson bound state to form a baryon with a heavy quark.

This is an Open Access article published by World Scientific Publishing Company. It is distributed under the terms of the Creative Commons Attribution 3.0 (CC-BY) License. Further distribution of this work is permitted, provided the original work is properly cited. 


\section{The Model}

We start from a chiral soliton model for light baryons. The major building block is the non-linear representation of the pesudoscalar mesons in form of the chiral field $U$. Rather than using higher derivative terms as in the Skyrme model ${ }^{6,7}$ to stabilize the soliton, here stabilization is achieved by coupling the pseudoscalar mesons to the vector mesons $\rho$ and $\omega .^{8-10}$ Though we utilize a three flavor model, the soliton profiles are embedded in the $S U(2)$ subgroup of isospin

$$
U_{0}(\boldsymbol{r})=\exp [\boldsymbol{\tau} \cdot \hat{\boldsymbol{r}} F(r)], \quad \omega_{\mu}^{(0)}(\boldsymbol{r})=\omega(r) g_{\mu 0}, \quad \boldsymbol{\rho}^{(0)}(\boldsymbol{r})=\boldsymbol{\tau} \times \hat{\boldsymbol{r}} \frac{G(r)}{r} .
$$

The profile functions $F(r), \omega(r)$ and $G(r)$ are determined by minimizing the classical energy, $E_{\mathrm{cl}}$ subject to boundary conditions that ensure unit winding number for the mapping of coordinate space into flavor space. Note that, on this classical level, the spatial components of the isoscalar field $\omega_{\mu}$ and the time components of isovector field $\rho_{\mu}$ are zero. We generate states with good baryon numbers by introducing ${ }^{a}$ and quantizing collective coordinates for the flavor orientation $A(t) \in S U(3)$

$$
U(\boldsymbol{r}, t)=A(t) U_{0}(\boldsymbol{r}) A^{\dagger}(t) \quad \text { and } \quad \boldsymbol{\tau} \cdot \boldsymbol{\rho}_{\mu}(\boldsymbol{r}, t)=A(t) \boldsymbol{\tau} \cdot \boldsymbol{\rho}_{\mu}^{(0)}(\boldsymbol{r}) A^{\dagger}(t) .
$$

Defining eight angular velocities $\Omega_{a}$

$$
\frac{i}{2} \sum_{a=1}^{8} \Omega_{a} \lambda_{a}=A^{\dagger}(t) \frac{d A(t)}{d t}
$$

enables a compact form for the collective coordinate Lagrange function that arises from the light meson fields

$$
L_{l}\left(\Omega_{a}\right)=-E_{\mathrm{cl}}+\frac{1}{2} \alpha^{2} \sum_{i=1}^{3} \Omega_{i}^{2}+\frac{1}{2} \beta^{2} \sum_{\alpha=4}^{7} \Omega_{\alpha}^{2}-\frac{\sqrt{3}}{2} \Omega_{8} .
$$

The last term is only linear in the time derivative and originates from the WessZumino-Witten action. ${ }^{12}$ The coefficients $\alpha^{2}$ and $\beta^{2}$ are radial integrals of the profile functions and represent moments of inertia for rotations in isospace and the strangeness subspace of flavor $S U(3)$, respectively. The form of the collective coordinate Lagrangian, Eq. (4) is generic to all chiral soliton models. The particular numerical values for the classical energy and the moments of inertia are, of course, subject to the particular model for which we will follow Ref. 10. The rotations also induce vector field components that vanish classically (see above). So far the collective coordinates only enter through their time derivatives; $A$ does not appear explicitly as we have not yet included $S U(3)$ flavor symmetry breaking contributions.

\footnotetext{
${ }^{a}$ Also profile functions are induced for the spatial components of $\omega_{\mu}$ and the time components of $\rho_{\mu} \cdot{ }^{10,11}$
} 


\section{Heavy Meson Bound States}

In the heavy flavor limit the pseudoscalar $(P)$ and vector meson $\left(Q_{\mu}\right)$ components become degenerate ${ }^{13}$ and must therefore be combined in a single multiplet $H=\frac{1}{2}\left(i \gamma_{5} P+\gamma^{\mu} Q_{\mu}\right)$. The Dirac spinor labels are subject to the heavy flavor transformation properties while the light flavor index of this multiplet $\left(P\right.$ and $Q_{\mu}$ are $S U(3)$ flavor spinors) is coupled to the light meson fields according to rules of chiral symmetry. This coupling to the soliton induces a potential for $P$ and $Q_{\mu}$ that allows for bound state solutions with energy eigenvalue $\omega$. Since the soliton configuration itself has non-zero orbital angular momentum the most strongly bound solution has a $\mathrm{P}$-wave structure in the pseudoscalar component

$$
\begin{aligned}
P & =\frac{\mathrm{e}^{i \omega t}}{\sqrt{4 \pi}} \Phi(r) \hat{\boldsymbol{r}} \cdot \hat{\boldsymbol{\tau}} \chi, \quad Q_{0}=\frac{\mathrm{e}^{i \omega t}}{\sqrt{4 \pi}} \Psi_{0}(r) \chi \\
Q_{i} & =\frac{\mathrm{e}^{i \omega t}}{\sqrt{4 \pi}}\left[i \Psi_{1}(r) \hat{r}_{i}+\frac{1}{2} \Psi_{2}(r) \epsilon_{i j k} \hat{r}_{j} \tau_{k}\right] \chi .
\end{aligned}
$$

where $\chi$ is a constant three component spinor. Since the coupling to the light mesons occurs via a soliton in the isospin subspace, only the first two components of $\chi$ are non-zero. The parameterization that emerges by left multiplication with $\hat{\boldsymbol{r}} \cdot \hat{\boldsymbol{\tau}}$ and, of course, has different profile functions, leads to the S-wave bound states.

Since the heavy meson fields are spinors in $S U(3)$ flavor space, they are also subject to collective flavor rotation from Eq. (2),

$$
P \rightarrow A(t) P \quad \text { and } \quad Q_{\mu} \rightarrow A(t) Q_{\mu}
$$

where the right hand sides contain the bound state profile functions. From this we get the collective coordinate Lagrange function from the heavy fields

$$
L_{h}\left(\Omega_{a}\right)=-\omega \chi^{\dagger} \chi+\frac{1}{2 \sqrt{3}} \chi^{\dagger} \Omega_{8} \chi+\rho \chi^{\dagger}\left(\Omega \cdot \frac{\boldsymbol{\tau}}{2}\right) \chi .
$$

The coefficient $\rho$ in the last term is a radial integral over the profile functions. ${ }^{14}$ Again, the flavor rotation matrix $A$ does not appear explicitly.

\section{Symmetry Breaking and Mass Formula}

So far we have not taken into account three flavor symmetry breaking as reflected by the different (current) quark masses of up, down and strange quarks, $m_{u}, m_{d}$ and $m_{s}$, respectively. While it is appropriate to work with $m_{u}=m_{d}$, the deviation $m_{s} \gg m_{u}, m_{d}$ is substantial and must be included. It is incorporated in the effective chiral Lagrangian by adding terms like

$$
\mathcal{L}_{\mathrm{sb}} \sim \frac{f_{\pi}^{2} m_{\pi}^{2}}{4} \operatorname{Tr}\left[\left(\begin{array}{lll}
1 & 0 & 0 \\
0 & 1 & 0 \\
0 & 0 & x
\end{array}\right)\left(U+U^{\dagger}-2\right)\right]+\cdots \quad \text { where } x \widehat{=} \frac{2 m_{s}}{m_{u}+m_{d}} \gg 1
$$


Eventually they describe different masses and decay constants of strange and nonstrange mesons. ${ }^{\mathrm{b}}$ Once the profile functions are substituted and the spatial integration is performed, symmetry breaking induces an explicit $A$ dependence in the collective coordinate Lagrange function

$$
L_{\mathrm{sb}}=-\frac{x}{2} \widetilde{\gamma}\left[1-D_{88}(A)\right] \quad \text { with } D_{a b}=\frac{1}{2} \operatorname{Tr}\left[\lambda_{a} A \lambda_{b} A^{\dagger}\right] .
$$

Again, $\widetilde{\gamma}$ is a radial integral ${ }^{\mathrm{c}}$ over all profile functions. ${ }^{5}$

Collecting pieces from Eqs. (2), (7) and (9) and Legendre transforming for the angular velocities via the right $S U(3)$ generators $R_{a}=\frac{\partial L}{\partial \Omega_{a}}$ yields the Hamilton operator whose eigenvalues are the baryon masses. This results in the mass formula

$$
\begin{aligned}
E= & E_{\mathrm{cl}}+\left(\frac{1}{\alpha^{2}}-\frac{1}{\beta^{2}}\right) \frac{r(r+1)}{2}+\frac{\epsilon(x)}{2 \beta^{2}}-\frac{3}{8 \beta^{2}}\left(1-\frac{N}{3}\right)^{2} \\
& +|\omega| N+\frac{\rho}{2 \alpha^{2}}[j(j+1)-r(r+1)] N,
\end{aligned}
$$

where $N$ counts the number of heavy quarks. Here $\epsilon(x)$ is the eigenvalue of $O_{\mathrm{sb}}=\sum_{a=1}^{8} R_{a}^{2}+x \beta \widetilde{\gamma}\left[1-D_{88}(A)\right]$ according to the Yabu-Ando approach ${ }^{15}$ subject to the constraint $R_{8}=\sqrt{3}\left(1-\frac{N}{3}\right) / 2$. For heavy baryons this constraint then requires diquark $S U(3)$ representations. Furthermore $j$ is the total spin of the consider baryon and $r(r+1)$ is the eigenvalue of $\sum_{i=1}^{3} R_{i}^{2}$. It is zero and one for the antisymmetric and the symmetric diquark wave-functions, respectively. ${ }^{\mathrm{d}}$ We stress that obtaining the eigenvalues $\epsilon(x)$ of the operator $O_{\mathrm{sb}}$ amounts to a non-perturbative treatment of symmetry breaking in the light flavor sector.

\section{Results}

All coefficients in the mass formula, Eq. (10) are determined from the soliton model calculation detailed in Refs. 10, 4 and 14. The only free parameter is the strength, $x$ of flavor symmetry breaking. It has been estimated ${ }^{16-18}$ from meson properties to be in the range $x \sim 20 . .30$. In Tables $1-3$ we list our predictions for the mass differences $^{\mathrm{e}}$ of the heavy baryons with the respect to the nucleon and the $\Lambda_{c, b}$ and compare them to empirical data. ${ }^{20}$ The mass differences within a given heavy quark sector is overestimated. For example $M_{\Omega_{c}}-M_{\Lambda_{c}}=463 \mathrm{MeV}$ for $x=25$, while the empirical value is $409 \mathrm{MeV}$. Further increase of $x$ worsens the picture. On the other hand, a sizable value $(x \sim 30)$ for the symmetry breaking is required for a good agreement for non-heavy baryons. ${ }^{10}$ The splitting between different heavy sectors is predicted on the low side: when compared to the nucleon, the $\Lambda_{c}$ and $\Lambda_{b}$ are about $100 \mathrm{MeV}$ and $300 \mathrm{MeV}$ too low, respectively. This is inherited from the heavy flavor

\footnotetext{
${ }^{\mathrm{b}}$ Symmetry breaking for the heavy mesons, proportional to e.g. $M_{B_{s}}^{2}-M_{B}^{2}$, is also included. For brevity the corresponding Lagrangian is not displayed here.

${ }^{\mathrm{c}}$ The notation is chosen to distinguish it from $\gamma=x \widetilde{\gamma}$ in the literature. ${ }^{2}$

${ }^{\mathrm{d}}$ Non-heavy baryons have $N=0$ and $r=j$.

${ }^{\mathrm{e}}$ We concentrate on mass differences to avoid ambiguities from the vacuum polarization energy. ${ }^{19}$
} 
Table 1. Predicted mass differences for the positive parity baryons with a single charm quark for two different strengths of $S U(3)$ symmetry breaking in comparison with experimental data (PDG). ${ }^{20}$ The mass differences with respect to the nucleon and the $\Lambda_{c}$ are denoted by $\Delta_{N}$ and $\Delta_{\Lambda_{c}}$, respectively.

\begin{tabular}{|c|c|c|c|c|c|c|c|}
\hline \multirow[b]{2}{*}{ Bary. } & \multirow[b]{2}{*}{$(I, j, r)$} & \multicolumn{2}{|c|}{$x=25$} & \multicolumn{2}{|c|}{$x=30$} & \multicolumn{2}{|c|}{ Data (PDG) } \\
\hline & & $\Delta_{N}$ & $\Delta_{\Lambda_{c}}$ & $\Delta_{N}$ & $\Delta_{\Lambda_{c}}$ & $\Delta_{N}$ & $\Delta_{\Lambda_{c}}$ \\
\hline$\Lambda_{c}$ & $(0,1 / 2,0)$ & 1230 & 0 & 1233 & 0 & 1347 & 0 \\
\hline$\Sigma_{c}$ & $(1,1 / 2,1)$ & 1423 & 193 & 1425 & 192 & 1515 & 168 \\
\hline$\Xi_{c}$ & $(1 / 2,1 / 2,0)$ & 1446 & 216 & 1486 & 253 & 1529 & 182 \\
\hline$\Omega_{c}$ & $(0,1 / 2,1)$ & 1693 & 463 & 1756 & 523 & 1756 & 409 \\
\hline$\Xi_{c}$ & $(1 / 2,1 / 2,1)$ & 1557 & 328 & 1588 & 355 & 1637 & 290 \\
\hline$\Sigma_{c}$ & $(1,3 / 2,1)$ & 1464 & 234 & 1466 & 233 & 1579 & 232 \\
\hline$\Xi_{c}$ & $(1 / 2,3 / 2,1)$ & 1598 & 369 & 1629 & 396 & 1706 & 359 \\
\hline$\Omega_{c}$ & $(0,3 / 2,1)$ & 1734 & 504 & 1797 & 564 & 1831 & 484 \\
\hline
\end{tabular}

Table 2. Same as Table 1 for the negative parity charmed baryons.

\begin{tabular}{|c|c|c|c|c|c|c|c|}
\hline \multirow[b]{2}{*}{ Bary. } & \multirow[b]{2}{*}{$(I, j, r)$} & \multicolumn{2}{|c|}{$x=25$} & \multicolumn{2}{|c|}{$x=30$} & \multicolumn{2}{|c|}{ Data (PDG) } \\
\hline & & $\Delta_{N}$ & $\Delta_{\Lambda_{c}}$ & $\Delta_{N}$ & $\Delta_{\Lambda_{c}}$ & $\Delta_{N}$ & $\Delta_{\Lambda_{c}}$ \\
\hline$\Lambda_{c}$ & $(0,1 / 2,0)$ & 1479 & 249 & 1482 & 249 & 1653 & 306 \\
\hline$\Sigma_{c}$ & $(1,1 / 2,1)$ & 1664 & 434 & 1666 & 433 & - & - \\
\hline$\Xi_{c}$ & $(1 / 2,1 / 2,0)$ & 1695 & 465 & 1735 & 502 & 1851 & 504 \\
\hline$\Omega_{c}$ & $(0,1 / 2,1)$ & 1934 & 704 & 1997 & 764 & - & - \\
\hline$\Xi_{c}$ & $(1 / 2,1 / 2,1)$ & 1798 & 569 & 1829 & 596 & - & - \\
\hline$\Sigma_{c}$ & $(1,3 / 2,1)$ & 1717 & 487 & 1719 & 486 & - & - \\
\hline$\Xi_{c}$ & $(1 / 2,3 / 2,1)$ & 1851 & 622 & 1882 & 649 & - & - \\
\hline$\Omega_{c}$ & $(0,3 / 2,1)$ & 1987 & 757 & 2050 & 817 & - & - \\
\hline
\end{tabular}

Table 3. Same as Table 1 for the positive parity bottom baryons. Here $\Delta_{\Lambda_{b}}$ is the mass difference with respect to $\Lambda_{b}$.

\begin{tabular}{|c|c|c|c|c|c|c|c|}
\hline \multirow[b]{2}{*}{ Bary. } & \multirow[b]{2}{*}{$(I, j, r)$} & \multicolumn{2}{|c|}{$x=25$} & \multicolumn{2}{|c|}{$x=30$} & \multicolumn{2}{|c|}{ Data (PDG) } \\
\hline & & $\Delta_{N}$ & $\Delta_{\Lambda_{c}}$ & $\Delta_{N}$ & $\Delta_{\Lambda_{c}}$ & $\Delta_{N}$ & $\Delta_{\Lambda_{c}}$ \\
\hline$\Lambda_{b}$ & $(0,1 / 2,0)$ & 4391 & 0 & 4394 & 0 & 4681 & 0 \\
\hline$\Sigma_{b}$ & $(1,1 / 2,1)$ & 4601 & 210 & 4603 & 209 & 4872 & 191 \\
\hline$\Xi_{b}$ & $(1 / 2,1 / 2,0)$ & 4608 & 216 & 4647 & 253 & 4855 & 174 \\
\hline$\Omega_{b}$ & $(0,1 / 2,1)$ & 4871 & 480 & 4935 & 540 & 5110 & 429 \\
\hline$\Xi_{b}$ & $(1 / 2,1 / 2,1)$ & 4736 & 345 & 4766 & 372 & - & - \\
\hline$\Sigma_{b}$ & $(1,3 / 2,1)$ & 4617 & 226 & 4619 & 225 & 4893 & 212 \\
\hline$\Xi_{b}$ & $(1 / 2,3 / 2,1)$ & 4751 & 360 & 4782 & 387 & 5006 & 325 \\
\hline$\Omega_{b}$ & $(0,3 / 2,1)$ & 4887 & 496 & 4950 & 556 & - & - \\
\hline
\end{tabular}

calculation which overestimates ${ }^{4}$ the binding energies in the sense that it is too close to the estimate from exact heavy flavor symmetry. This can also be seen from the parity splitting which is underestimated by about $50 \mathrm{MeV}$ (it vanishes in the heavy limit). Together with the effect of $S U(3)$ symmetry breaking the overestimated binding combines to acceptable agreement for the mass differences between the 
double strange baryons $\Omega_{c}$ and $\Omega_{b}$ and the nucleon, at least for $x=30$. It has been argued $^{14}$ that kinematical corrections (e.g. substituting the reduced mass in the bound state problem) due to the soliton not being infinitely heavy change the predicted bound state energies appropriately.

For $j=1 / 2$ and positive parity the observed mass difference between $\Sigma$ and $\Xi$ decreases and even changes sign when the heaviest flavor turns from strange via charm to bottom: $M_{\Xi}-M_{\Sigma}=125,14,-17 \mathrm{MeV}$. This is (partially) reflected by our calculation, e.g. for $x=25$ we find the mass differences 101,23 and $6 \mathrm{MeV}$. Since the hyperfine splitting only has a moderate effect, we find a similar scenario for the negative parity channel and it will be interesting to compare it with future data.

\section{Summary}

We have presented model predictions for the spectrum of baryons with a single heavy quark. Our analysis culminates in a single mass formula for the spectrum. The ingredients of the mass formula are the binding energies of the heavy meson, the flavor symmetry breaking among the non-heavy flavors and the hyperfine splitting. In these aspects our study builds on and extends an earlier chiral soliton model approach $^{21}$ on heavy baryons with strangeness. We stress that, except for a moderate uncertainty of the light symmetry breaking strength, all parameters in our mass formula are obtained from a single model Lagrangian. Though not presented here, we recall that this model also predicts ${ }^{10}$ the spectrum and static properties of light baryons with reasonable agreement to empirical data.

\section{Acknowledgments}

One of us (HW) is grateful to the organizers for providing this worthwhile conference. This work supported in parts by the NRF under grant 77454 .

\section{References}

1. R. Aaij et al., Phys. Rev. Lett. 115, 072001 (2015).

2. H. Weigel, Lect. Notes Phys. 743, 1 (2008).

3. J. Schechter and A. Subbaraman, Phys. Rev. D 48, 332 (1993).

4. J. Schechter, A. Subbaraman, S. Vaidya and H. Weigel, Nucl. Phys. A 590, 655 (1995).

5. J. P. Blanckenberg and H. Weigel, Heavy Baryons with Strangeness in a Soliton Model, arXiv:1505.06655, (2015).

6. T. H. R. Skyrme, Proc. Roy. Soc. Lond. A 260, 127 (1961).

7. G. S. Adkins, C. R. Nappi and E. Witten, Nucl. Phys. B 228, 552 (1983).

8. P. Jain, R. Johnson, Ulf-G. Meißner, N. W. Park and J. Schechter, Phys. Rev. D 37, 3252 (1988).

9. B. Schwesinger, H. Weigel, G. Holzwarth and A. Hayashi, Phys. Rept. 173, 173 (1989).

10. N. W. Park and H. Weigel, Nucl. Phys. A 541, 453 (1992).

11. Ulf-G. Meißner, N. Kaiser and W. Weise, Nucl. Phys. A 466, 685 (1987).

12. E. Witten, Nucl. Phys. B 223, 422, 433 (1983).

13. M. Neubert, Phys. Rept. 245, 259 (1994). 
14. M. Harada, A. Qamar, F. Sannino, J. Schechter and H. Weigel, Nucl. Phys. A 625, 789 (1997).

15. H. Yabu and K. Ando, Nucl. Phys. B 301, 601 (1988).

16. J. Gasser and H. Leutwyler, Phys. Rept. 87, 77 (1982).

17. J. Schechter, A. Subbaraman and H. Weigel, Phys. Rev. D 48, 339 (1993).

18. M. Harada and J. Schechter, Phys. Rev. D 54, 3394 (1996).

19. F. Meier and H. Walliser, Phys. Rept. 289, 383 (1997).

20. K. A. Olive et al., Chin. Phys. C 38, 090001 (2014).

21. A. Momen, J. Schechter and A. Subbaraman, Phys. Rev. D 49, 5970 (1994). 